\title{
Physical activity equivalents for takeaway food: a new method for nutritional labelling
}

\author{
I.G. Davies, L. Stevenson and M.J. Reeves \\ School of Education, Leisure \& Sport Studies, Liverpool John Moores University, Liverpool, L17 6BD
}

Consumption of takeaway food is a public health concern, especially in relation to obesity and related diseases ${ }^{(1)}$. While the UK has postulated a standardised traffic light labelling system to address this problem ${ }^{(2)}$ the evidence remains equivocal ${ }^{(3)}$ and many takeaway establishments have not participated. Consumers generally misjudge the energy they consume and find calorie information difficult to interpret. This may be related to how caloric information is presented and evidence suggests that physical activity equivalents are effective in reducing point of purchase energy ${ }^{(4,5)}$. Therefore, the present study introduces the use of physical activity as an alternative method of presenting energy for takeaway food. Samples of takeaway meals $(n=717)$ were collected from small independent establishments and analysed for total energy by an accredited public analyst laboratory and converted to jogging hours using metabolic equivalent (METS) ${ }^{(6)}$. Meal categories were compared using a one way ANOVA. Results revealed a high variability in mean hours of general jogging with significant differences between meal categories $(p<0 \cdot 001$, Figure 1$)$. Pizza meals were the highest in jogging hours with up to $5 \mathrm{~h}$ per meal for females.
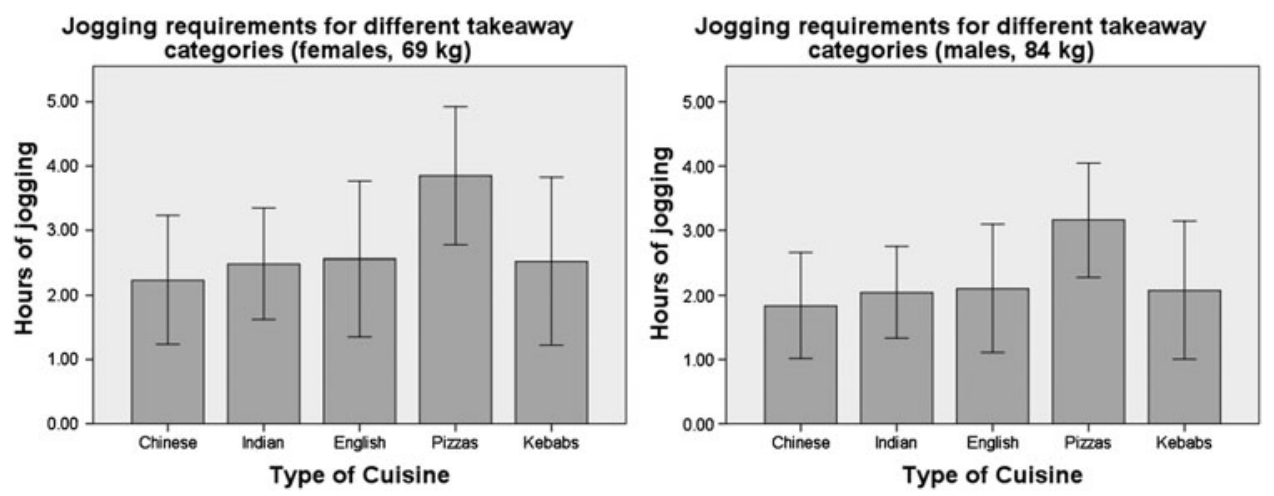

Figure 1. Mean hours of jogging for both males and females (means \pm SD)

This current study presents evidence on takeaway food, not only showing high levels of physical activity requirements for energy expenditure but also offers an alternative method of presenting caloric information to consumers. Previous studies have found this concept to be effective in the USA ${ }^{(4,5)}$. Considering the lack of conclusive evidence on nutritional labelling in various formats, physical activity equivalents should be considered for future research to reduce energy intake in the fast and takeaway food industry where meals are excessively high in energy.

1. Jaworowska A, Blackham T, Davies I.G., et al. (2013). Nutrition Reviews 71 (5), 310-18.

2. Dept. of Health (2013). https://www.gov.uk/government/policies/reducing-obesity-and-improving-diet

3. Dodds P, Wolfend L, Chapman K., et al. (2014). Appetite 73, 23-30.

4. Bleich S.N., Herring B.J., Flagg D.D., et al. (2013). Am J Public Health 104 (12), 2417-24.

5. James A, Adams-Huet B, \& Shah M (2014). Am J of Health Promotion (published head of press).

6. Ainsworth B.E., Haskell W.L., Whitt M.C., et al. (2000). Medicine and science in sports and exercise 32 9; SUPP/1: S498-S504. 